\title{
Novel Designer Plastic Trumpet Bells for Brass Instruments
}

\author{
David Gibson \\ Birmingham City University \\ Faculty of Computing, Engineering \\ and the Built Environment \\ Millennium Point, Curzon St, \\ Birmingham, B4 7XG, United Kingdom \\ david.gibson@bcu.ac.uk
}

\begin{abstract}
It is proposed that by using computing analysis software and 3D fabrication techniques, low cost plastic trumpet bells could be produced for different music genres by altering their timbres. These may be attached to the trumpet in a similar manner to that of a tuning bell trumpet. The novel bells are designed to eliminate or enhance particular harmonics to change their brightness by altering the thickness of material, thus altering the impact of the vibrations to the sound column within it. This initial paper focuses on a comparison between the natural resonances of a current trumpet brass bell and various modified plastic bells using simulation software. Various designs are presented that show the resulting changes of the resonance harmonic mode frequencies and deformation amplitudes for Acrylonitrile butadiene styrene (ABS) can be shifted toward that of brass depending on the thickness of the material thus paving the way for designed plastic trumpet bells with similar tones.
\end{abstract}

3D Plastic instruments. Bell vibrations. Trumpet bell harmonics.

\section{INTRODUCTION}

Brass trumpets are known to produce different timbres described as sounding 'bright' or 'dark' by musicians due to the tones produced. According to research this is due to the design parameters of the bell and lead pipe (Buick et al. 2002). Depending on the type of music being played, it would be helpful to the musician to be able to change the tone without having to use an alternative instrument. An example would be the need for a deep dark sound for a Mahler symphony whereas a brighter sound may be preferred for light music or jazz.

In general, the development of trumpet bells has been based on evolution rather than acoustic design and only recently have the mechanisms through which the bell plays a part in producing changes in tone been fully understood.

Recently, developments in instrument design and fabrication techniques have resulted in the development of plastic trombones and trumpets specifically for learners in Schools. Examples include the pBone - 2011, tromba Jazzbone, pTrumpet - 2014, Tiger trumpet, Allora Aere lightweight and tromba trumpet. These are commercially available, their components being injection moulded for mass production. The sound produced by these instruments appears similar to their brass counterparts although lacking in perceived 'brightness' or overtones. Other plastic 3D printed instruments have been manufactured with some success (Zoran 2011), demonstrating that this fabrication technique may become more widespread in custom instrument manufacture. A plastic printable trumpet design has also been made available on the internet (LtDan 2015) although the author states that the quality of sound produced is, in general, poor.

The use of materials other than brass for instrument manufacture and their effect on the radiated sound spectrum have been debated for many years. Smith (1978), Schilke (n.d.), and Pyle (1997) all state that the sound spectrum produced is altered due to material type, thickness and fabrication methods. There is agreement that thinner (brass) bells alter the vibrations contained within them and accentuate the higher frequencies. However, not all agree that the material type significantly contributes to the tone but rather the thickness of the material. However, almost a decade after Smith originally states that thickness mattered, he concludes that the bell thickness does not have a significant effect on the far field sound, only that in the near field for the player who in turn would alter the timbre produced (Smith 1986). 
Nachtmann, Kausel \& Mayer (2007) consider whether there is a correlation between bell vibrations and radiated sound. This is to differentiate between the instrumentalist's views stated above and the theorist view; that the resonant air column significantly contributes to the sound and the vibrations only play a minor part in the tone. This difference in opinion as to why the sound may alter was attributed to the earlier subjective methods used for testing. However, their findings using sand bags based upon work by Moore et al. (2005) to dampen an artificially excited bell demonstrates that vibrations do cause significant changes to the sound spectrum which can also be detected by listeners in the far field.

Kausal et al. (2010) demonstrate theoretically that the radiated sound is affected by the vibrations of the instrument coupled to the internal sound field. They show that vibrations are most significant in the flare of the bell and not in the cylindrical pipes and that the primary effects are constrained to the first $15 \mathrm{~cm}$ of the bell section.

Further investigation by Kausel et al. (2015) on axial vibrations of the bell and their contribution to the tones produced conclude that damping the bell vibrations can contribute to impedance changes and thus the sound (tone) produced. Moore (2015) applied Kausel's model and verified through experimentation that the axial vibrations do affect the sound generated with elliptical vibrations less so.

By understanding the effect the vibrations have on the structural modes of the bell it is possible to investigate how the acoustic spectrum may be influenced. Although Smith concluded that the differences in tone produced due to material and thickness had little effect in terms of sound to the player his research was based upon trombone bells and was subjective in nature even though certain harmonics were increased by up to $2 \mathrm{~dB}$. The work of Kausel and Moore suggests otherwise, with differences in the power contained in overtones exceeding $6 \mathrm{~dB}$ being stated.

This initial paper investigates through simulation of natural bell resonances (vibrations) whether a plastic bell may be designed to emulate a brass equivalent.

\section{EXPERIMENTAL METHODOLOGY}

The concept of producing a plastic bell that can emulate a brass bell requires study of the components that affect the axial air column within the bell of the instrument. This behaves as the amplifier of the system but also contributes to the sound spectrum. As stated above both axial and elliptical vibrations have an influence on the way the flare of the bell vibrates and this adds distortions to the air column giving the trumpet its distinctive sound. The placement of braces, the stiffness of the solder joint along the axis of the bell as well as the homogeneity of the thickness of the material used all add to the complexity of the sound produced. With hand-crafted instruments these change considerably and hence no two trumpets sound the same. By manufacturing plastic bells the issue of repeatability, seam problems and mounting (based on a tuning bell trumpet), will be minimised or eliminated altogether.

An example of a mock-up of a plastic bell on a Schilke B1 trumpet is shown in Figure 1. The aim is to be able to fabricate these bells using professional 3D printing companies who can produce them at low cost compared to a brass bell. Furthermore, by changing the design parameters it may be possible to alter the timbre. The musician may therefore carry a number of bells for playing purposes. The bells will also be durable especially if knocked which unlike brass instruments will not be dented which usually results in a high cost of repair.

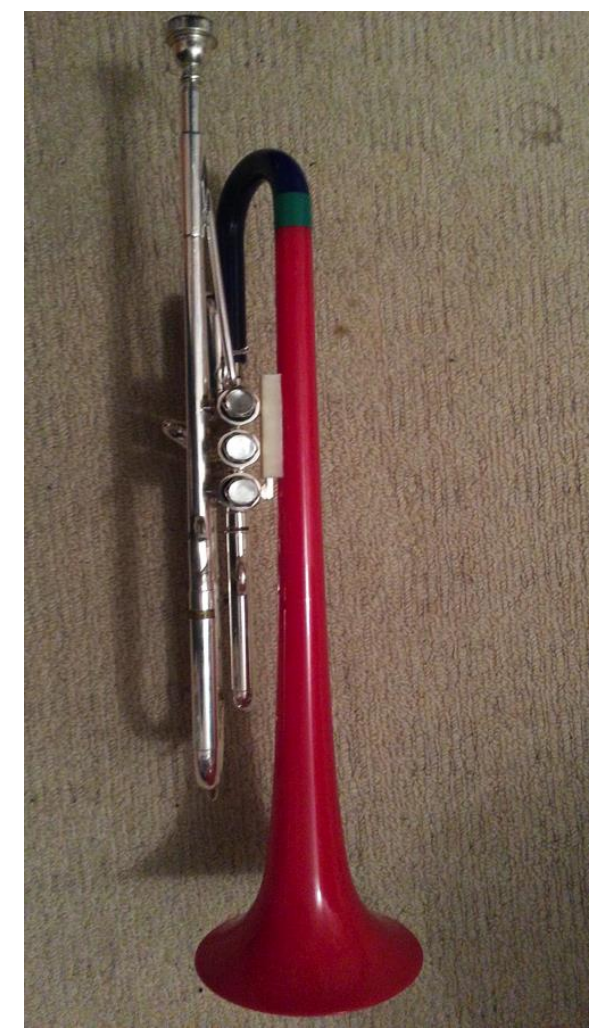

Figure 1: Mock-up of plastic bell on tuning bell trumpet

The initial investigation will focus on finite element analysis of the natural resonances of a trumpet bell. This is performed using ANSYS, an engineering design software package, to simulate and measure the natural vibrations and hence resonance frequencies produced. 
In this first stage of research a typical brass student trumpet bell has been scanned using GEOPAK (part of MiCAT) to produce a suitable CAD drawing. The bell is from a Corton student model. As it a student instrument the thickness is slightly greater than a professional trumpet. However, for the purposes of initial simulations this may be more appropriate as there will be fewer overtones produced (trumpet players who want a bright sound usually choose a thinner bell with material that produces higher resonances). This means that the ABS material may be better matched as its thickness is increased as it will not need to produce higher overtones.

The simulations of the trumpet bell design are undertaken using generic brass and ABS material models. The values of Young's modulus and density are given in Table 1.

Table 1: Material specifications

\begin{tabular}{|l|l|l|}
\hline \multirow{2}{*}{ Material } & \multicolumn{2}{c|}{ Specifications } \\
\cline { 2 - 3 } & $\begin{array}{l}\text { Young's } \\
\text { Modulus (Pa) }\end{array}$ & $\begin{array}{l}\text { Density } \\
\left(\mathrm{kgm}^{-3}\right)\end{array}$ \\
\hline Brass & $1 e^{11}$ & 8250 \\
\hline$A B S$ & $2 e^{9}$ & 1100 \\
\hline
\end{tabular}

For simulation purposes the thin end of the bell is anchored and the natural vibrations are measured across all frequencies.

The particular bell used has a wire rim which will influence the vibrations as detailed by Kausel et al. (2015). However, for the initial purpose of exploring the behaviour of the bell this has been copied as part of the bell profile (although the original material type is different from the brass and may influence the vibrations). The added complexity of the rim may be overcome in future by using a rimless bellas rimless plastic is less fragile than a brass equivalent (an example is the pTrumpet bell modelled on a Bach profile but rimless) but for the purpose of the simulations it has been left in.

As the plastic bell design is meant to emulate the brass bell responses the axial and elliptical vibrations between the two materials are investigated. Although according to Kausel the elliptical vibration modes do not significantly affect the far field sound it is useful to compare the deformities both axially and elliptically for comparison between the materials.

To investigate the initial effect, 3D FE simulations of thickness from 0.75 to $3.00 \mathrm{~mm}$ are run. The brass bell simulation is used as the benchmark and comparisons made with the plastic bell. Details of the bell specifications are given in Table 2 and thickness of the plastic bell in Table 3. Separate CAD drawings are produced for each using Catia V5 package.
Table 2: Bell specifications

\begin{tabular}{|l|l|l|l|}
\hline \multirow{2}{*}{ Bell type } & \multicolumn{3}{|c|}{ Bell Specifications } \\
\cline { 2 - 4 } & Rim diameter & Thickness & Length \\
\hline Brass & $125 \mathrm{~mm}, 47 / 8 "$ & $0.75 \mathrm{~mm}$ & $400 \mathrm{~mm}$ \\
\hline
\end{tabular}

As the comparison of vibrations within the bell is of interest the type of flare is not considered relevant to the measurements taken.

Table 3: Simulated bell variations

\begin{tabular}{|l|l|l|l|}
\hline \multirow{2}{*}{ Bell type } & \multicolumn{3}{|c|}{ Bell variations } \\
\cline { 2 - 4 } & Material & Bell Thickness $(\mathrm{mm})$ & Label \\
\hline Rim & Brass & 0.75 & $R 1$ \\
\hline $\operatorname{Rim}$ & $A B S$ & 0.75 & $R 2$ \\
\hline $\operatorname{Rim}$ & $A B S$ & 1.5 & $R 3$ \\
\hline $\operatorname{Rim}$ & $A B S$ & 2.25 & $R 4$ \\
\hline $\operatorname{Rim}$ & $A B S$ & 3.00 & $R 5$ \\
\hline
\end{tabular}

\section{SIMULATION RESULTS AND DISCUSSION}

To investigate the vibrations based on different thicknesses of material, comparison is made between the magnitudes of the deformations. Of interest is whether the change is proportional to thickness and if the deformations can occur at similar frequencies to the brass bell.

Results are shown for elliptical and axial vibrations with peak amplitudes at the flare of the bell. The amplitudes of the vibrations are shown on the left side of the plot with the largest deformation in red, smallest in dark blue. The frequency at which the deformation occurs is given in the top left of the plot.

The $\mathrm{Bb}$ trumpet produces standing waves at resonant frequencies that follow a harmonic sequence from F3 sharp; $185 \mathrm{~Hz}$ to $\mathrm{C} 6 ; 1046.5 \mathrm{~Hz}$. The deformations that occur within the harmonic sequence will have an effect on the behaviour of the wave that will give rise to the tone associated with the bell (although higher modes will also have some impact).

Examples of both elliptical and axial deformations are shown in Figures 2 to 5 . A total of 25 modes were simulated for each bell type. There are similarities in the mode shape but at different frequencies. This was similar for all modes for each type of bell. 


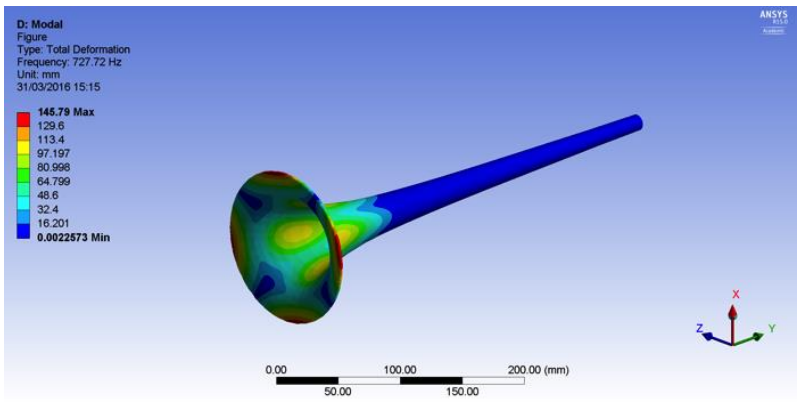

Figure 2: R1, Mode 6. Brass, rim, $0.75 \mathrm{~mm}$

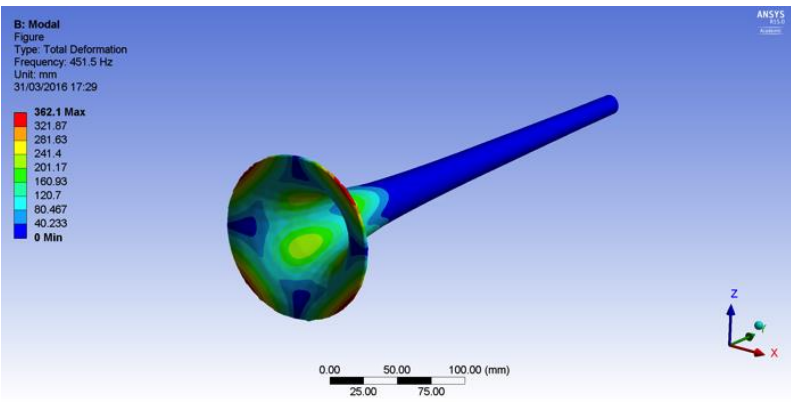

Figure 3: R3, Mode 6, $A B S$, rim, $1.5 \mathrm{~mm}$

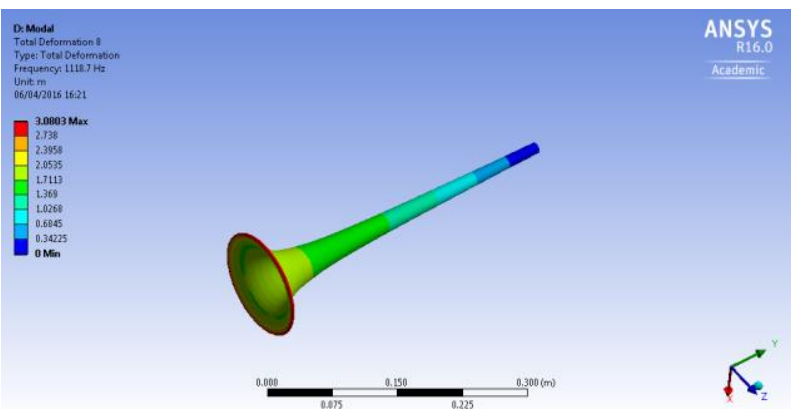

Figure 4:R1, Mode 8, Brass, rim, $0.75 \mathrm{~mm}$

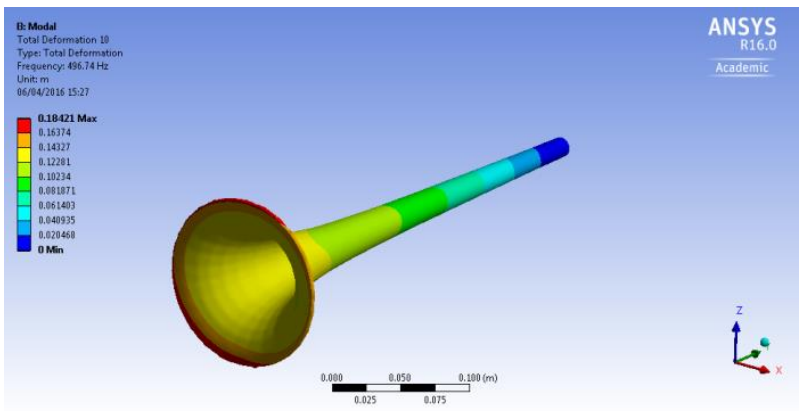

Figure 5: R3, Mode 10, $A B S$, rim, $1.5 \mathrm{~mm}$

The simulation results show that there is some correlation between the deformation amplitudes and frequency when increasing the thickness of the material. By thickening the ABS bell the mode frequencies for each increases whilst the deformation is reduced. The increase in thickness alters the rigidity of the bell therefore vibrations are further damped.

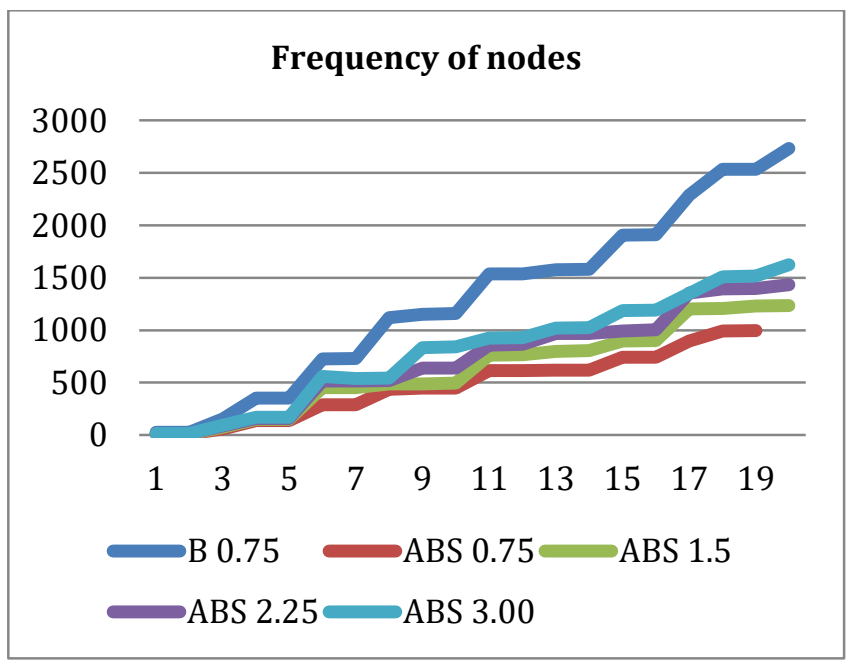

Figure 6: Mode frequencies $(\mathrm{Hz})$

In order to emulate the brass bell it is desirable for the plastic bell deformations to occur at similar mode frequencies. Due to the nature of the plastic being a 'softer' material it is expected that the resonances will be lower. The simulations clearly demonstrate this with frequency values of less than half that of the brass bell at $0.75 \mathrm{~mm}$ with an increase to approximately $60 \%$ at $3.00 \mathrm{~mm}$ (Figure 6).

Elliptical deformation decreased in comparison to the brass bell up to mode 12 as the thickness was increased (Figure 7). Axial deformations beyond mode 7 showed unpredictable behaviour as did the elliptical vibrations after mode 12 although again there was a tendency for the deformations to reduce. This is at the top range of the Bb trumpet and the influence this has, will need further investigation.

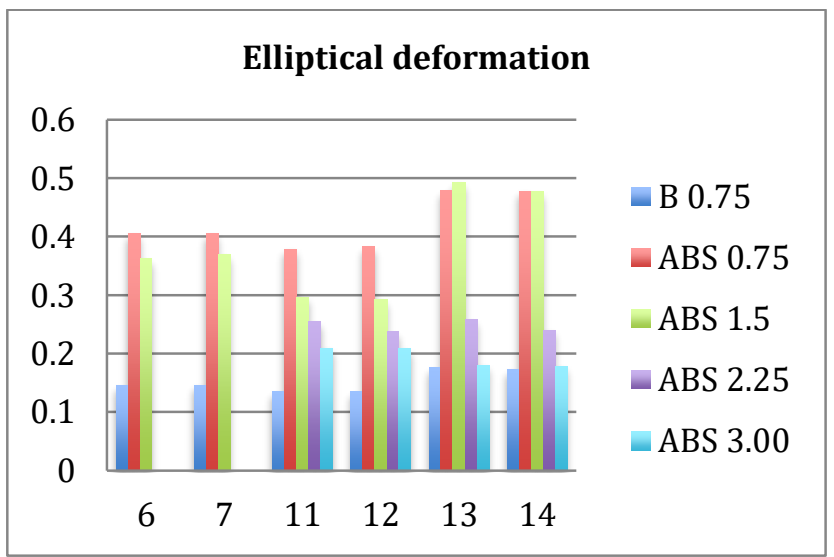

Figure 7: Elliptical deformations $727 \mathrm{~Hz}$ to $1907 \mathrm{~Hz}$ 


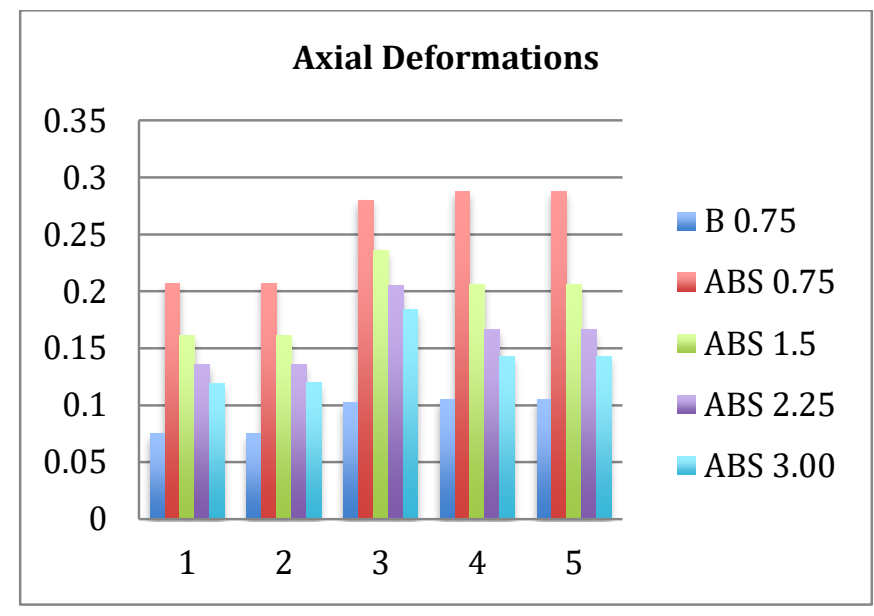

Figure 8: Axial deformations $26 \mathrm{~Hz}$ to $325 \mathrm{~Hz}$

\section{CONCLUSION}

The discussion presented here has highlighted the need to consider material thickness in the design of novel plastic bells for trumpets. Thickness and material type influence the axial vibrations that in turn alter the timbre as stated by Kausel. The initial simulations presented here show that the vibrations within the bell occur at similar nodes in the flare with reducing amplitudes to increased thickness of the material for lower modes. If an ABS bell is made thick enough then the vibrations begin to approach that of a thinner brass bell. However, further simulations are required to estimate what the optimum thickness should be using more accurate model parameters. Bells with a thickness of greater than three or four millimetres may become unwieldy as they get heavier than their brass counterparts! Different timbres will result from the change in thickness. Bells will need to be fabricated and timbre measured to determine how well the bell performs in the far field. Investigation of the repeatability of printing will also be necessary, an issue that has plagued manufacturers. Mass production using moulding techniques may facilitate repeatability. This will result in a new low cost fabricated bell.

\section{ACKNOWLEDGEMENTS}

I would like to thank Matthew King for his support in running the simulations and modifying the CAD drawings.

\section{REFERENCES}

Buick, J. M., Kemp, J., Sharp, D. B., van Walstijn, M., Campbell, D. M., and Smith, R. A., (2002)
Distinguishing between similar tubular objects using pulse reflectometry: a study of trumpet and cornet leadpipes, Measurement Science Technology, 13 pp.750-757.

Kausel, W., Zietlow, D. W.., and Moore, T., (2010) Influence of wall vibrations on the sound of brass wind instruments, Journal of Acoustical Society of America, vol. 128, pp.3161-3174.

Kausel, W., Chatziioannou, V., Moore, T., Gorrman, B. R., and Rokni, M., (2015) Axial vibrations of brass wind instrument bells and their acoustical influence: Theory and simulations, Journal of Acoustical Society of America, vol. 137, pp.3149-3162.

LtDan. http://www.thingiverse.com/thing:662115 (retrieved 18 March 2016).

Moore, T., Shirley, E. T., Codrey, I. E., and Daniels, A. E. (2005) The effects of bell vibrations on the sound of the modern trumpet, Acta Acustica united with Acustica vol. 91, pp.578-589.

Moore, T., Gorrman, B. R., Rokni, M., Kausel, W., and Chatziioannou, V. (2015) Axial vibrations of brass wind instrument bells and their acoustical influence: Experiments, Journal of Acoustical Society of America, vol. 138, pp.1233-1240.

Nachtmann, G., Kausel, W., and Mayer, A. (2007) Bell vibrations and radiated sound of brass instruments - Is there an audible correlation? $3^{\text {rd }}$ Congress of the Alps Adira Acoustics Association, Graz, Austria, 27-28 September 2007.

Pyle, R. W. (1997) How brass instruments are built: Art, craft, perhaps even science, Journal of Acoustical Society of America, vol 101, 3056.

Schilke, R. O. (n.d.), http:/www.dallasmusic.org/schilke/Brass\%20Clinic. html (retrieved 21 March 2016).

Smith, R.A., (1978) Recent developments in trumpet design, International Trumpet Guild Journal vol 3, pp.1-8.

Smith, R. A. (1986) The effect of material in brass instruments; a review, Proceedings of The Institute of Acoustics, vol 8, Part 1, pp. 91-96.

Zoran, A. (2011) The 3D Printed Flute: Digital Fabrication and Design of Musical Instruments, Journal of New Music Research, 40:4, pp.379-387. 